\title{
Free Vibration of Laminated Composite Hypar Shell Roofs with Cutouts
}

\author{
Sarmila Sahoo \\ Department of Civil Engineering, Meghnad Saha Institute of Technology, Kolkata 700107, India \\ Correspondence should be addressed to Sarmila Sahoo, sarmila_ju@yahoo.com
}

Received 6 May 2011; Revised 20 September 2011; Accepted 20 September 2011

Academic Editor: K. M. Liew

Copyright () 2011 Sarmila Sahoo. This is an open access article distributed under the Creative Commons Attribution License, which permits unrestricted use, distribution, and reproduction in any medium, provided the original work is properly cited.

Use of laminated composites in civil engineering structural components including shell roofs is increasing day by day due to their light weight, high specific strength, and stiffness properties. In the present paper, laminated composite hypar shell (hyperbolic paraboloidal shells bounded by straight edges) roofs with cutouts are analyzed for their free vibration characteristics using finite element method. An eight-noded curved shell element is used for modeling the shell. Specific numerical problems of earlier investigators are solved to compare their results with the present formulation. A number of problems are further solved where the size of the cutouts and their positions with respect to the shell centre are varied for different edge constraints. The results are furnished in the form of figures and tables. The results are examined thoroughly to arrive at some meaningful conclusions useful to designers.

\section{Introduction}

Different shell forms are common in civil engineering applications. Among the shell forms used as roofing units, the skewed hypars (hyperbolic paraboloidal shells bounded by straight edges) have a special position because these architecturally pleasant forms may be cast and fabricated conveniently being doubly ruled surfaces. Hypar shell is preferred in many places, particularly in medical, chemical and food processing industries where entry of north light is desirable. Application of hypars in these industries often necessitates provision of cutouts for the passage of light, to provide accessibility to other parts of the structure, for venting and also sometimes for alteration of the resonant frequency. A comprehensive idea about the static and free vibration characteristics of such shell roofs with cutouts is essential for a designer for successfully applying these forms. Moreover, nowadays researchers are emphasizing more on laminated composite shells realizing the strength and stiffness potentials of this advanced material.

The free vibration of composite as well as isotropic plate with cutout was studied by different researchers from time to time. Reddy [2] investigated large amplitude flexural vibration of composite plate with cutout. Later in 1989,
Malhotra et al. [3] studied free vibration of composite plate with cutout for different boundary conditions. One of the early reports on free vibration of curved panels with cutout was due to Sivasubramonian et al. [4]. They analysed the effect of cutouts on the natural frequencies of plates with some classical boundary conditions. The plate had a curvature in one direction and was straight in the other. The effect of fibre orientation and size of cutout on natural frequency on orthotropic square plates with square cutout was studied using Rayleigh-Ritz method. Later Sivakumar et al. [5], Rossi [6], Huang and Sakiyama [7], and Hota and Padhi [8] studied free vibration of plate with various cutout geometries. Chakravorty et al. [1] analyzed the effect of concentric cutout on different shell options. Again in 1999, Sivasubramonian et al. [9] studied the effect of curvature and cutouts on square panels with different boundary conditions. The size of the cutout (symmetrically located) as well as curvature of the panels is varied. Hota and Chakravorty [10] published useful information about free vibration of stiffened conoidal shell roofs with cutout. Later Nanda and Bandyopadhyay [11] studied the effect of different parametric variation on free vibration of cylindrical shell with cutout.

A scrutiny of the literature on vibration of shell panels with cutout indicates that there is enough scope of research 


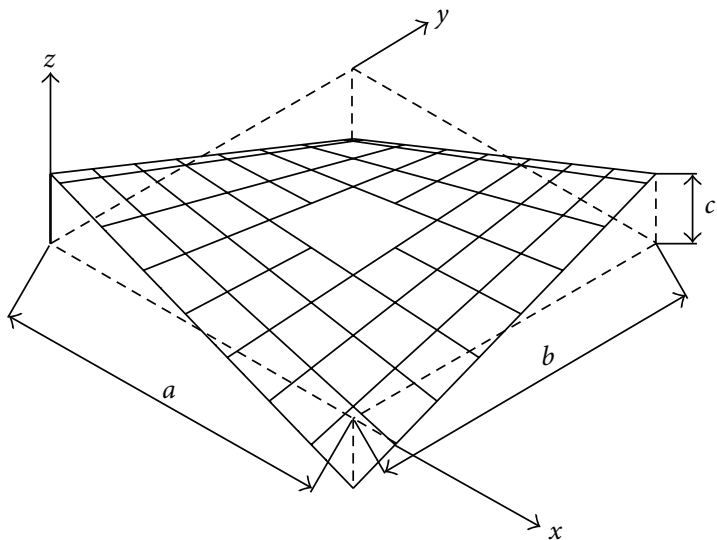

FIGURE 1: Surface of a hypar shell with cutout. Surface equation $z=$ $(4 c / a b)(x-a / 2)(y-b / 2)$

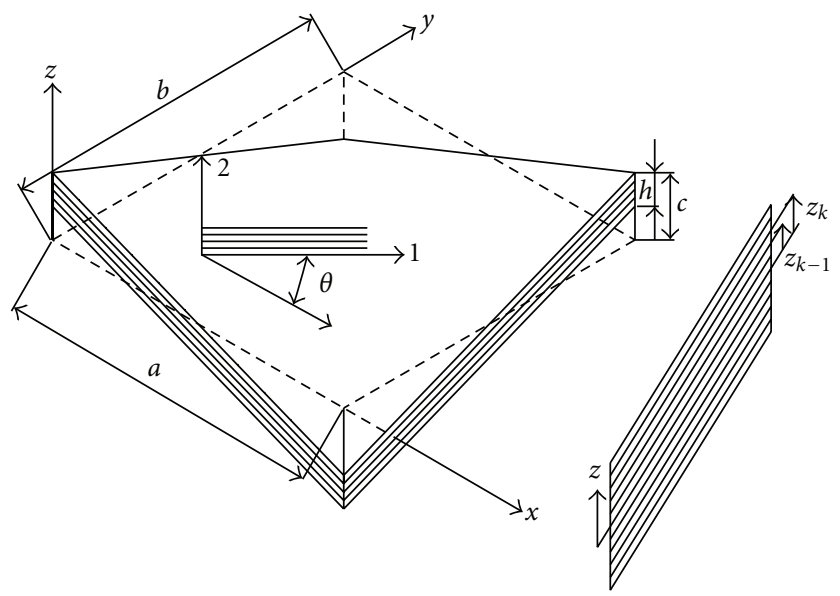

FIgURE 2: Laminations in skewed hypar shell.

to be carried out on the hypar shell. Although Chakravorty et al. [1] did a few studies about hypar shell with concentric cutout, many practically important aspects are yet to be addressed. In the present paper the free vibration of hypar shell with cutouts (Figure 1) is studied considering different boundary conditions. The variation of fundamental frequency due to change in eccentricity of cutout along $x$ and $y$ direction is also considered.

\section{Governing Equations}

A laminated composite hypar shell (Figure 2) of uniform thickness $h$ and twist radius of curvature $R_{x y}$ is considered. Keeping the total thickness same, the thickness may consist of any number of thin laminae each of which may be arbitrarily oriented at an angle $\theta$ with reference to the $x$-axis of the coordinate system. The constitutive equations for the shell are given by (a list of notations is already given)

$$
\{F\}=[D]\{\varepsilon\},
$$

where

$$
\begin{aligned}
& \{F\}=\left\{\begin{array}{lllllllll}
N_{x} & N_{y} & N_{x y} & M_{x} & M_{y} & M_{x y} & Q_{x} & Q_{y}
\end{array}\right\}^{T}, \\
& {[D]=\left[\begin{array}{llllllllll}
A_{11} & A_{12} & A_{16} & B_{11} & B_{12} & B_{16} & 0 & 0 \\
A_{12} & A_{22} & A_{26} & B_{12} & B_{22} & B_{26} & 0 & 0 \\
A_{16} & A_{26} & A_{66} & B_{16} & B_{26} & B_{66} & 0 & 0 \\
B_{11} & B_{12} & B_{16} & D_{11} & D_{12} & D_{16} & 0 & 0 \\
B_{12} & B_{22} & B_{26} & D_{12} & D_{22} & D_{26} & 0 & 0 \\
B_{16} & B_{26} & B_{66} & D_{16} & D_{26} & D_{66} & 0 & 0 \\
0 & 0 & 0 & 0 & 0 & 0 & S_{11} & S_{12} \\
0 & 0 & 0 & 0 & 0 & 0 & S_{12} & S_{22}
\end{array}\right],} \\
& \{\varepsilon\}=\left\{\begin{array}{llllllll}
\varepsilon_{x}^{0} & \varepsilon_{y}^{0} & \gamma_{x y}^{0} & k_{x} & k_{y} & k_{x y} & \gamma_{x z}^{0} & \left.\gamma_{y z}^{0}\right\}^{T} .
\end{array}\right.
\end{aligned}
$$

The force and moment resultants are expressed as

$$
\left\{\begin{array}{c}
N_{x} \\
N_{y} \\
N_{x y} \\
M_{x} \\
M_{y} \\
M_{x y} \\
Q_{x} \\
Q_{y}
\end{array}\right\}=\int_{-h / 2}^{h / 2}\left\{\begin{array}{c}
\sigma_{x} d z \\
\sigma_{y} d z \\
\tau_{x y} d z \\
\sigma_{x} z d z \\
\sigma_{y} z d z \\
\tau_{x y} z d z \\
\tau_{x z} d z \\
\tau_{y z} d z
\end{array}\right\} .
$$

The stiffness coefficients are functions of Young's moduli, shear moduli, and the Poisson's ratio of the laminates. They also depend on the angle which the individual lamina of a laminate makes with the global $x$-axis. The detailed expressions of the elements of the elasticity matrix are available in several references including Qatu [12] and Vasiliev et al. [13].

The stiffness coefficients are defined as

$$
\begin{gathered}
A_{i j}=\sum_{k=1}^{\mathrm{np}}\left(Q_{i j}\right)_{k}\left(z_{k}-z_{k-1}\right), \\
B_{i j}=\frac{1}{2} \sum_{k=1}^{\mathrm{np}}\left(Q_{i j}\right)_{k}\left(z_{k}^{2}-z_{k-1}^{2}\right), \\
D_{i j}=\frac{1}{3} \sum_{k=1}^{\mathrm{np}}\left(Q_{i j}\right)_{k}\left(z_{k}^{3}-z_{k-1}^{3}\right) \quad i, j=1,2,6, \\
S_{i j}=\sum_{k=1}^{\mathrm{np}} F_{i} F_{j}\left(G_{i j}\right)_{k}\left(z_{k}-z_{k-1}\right) \quad i, j=1,2,
\end{gathered}
$$

where $Q_{i j}$ are elements of the off-axis elastic constant matrix which is given by

$$
\left[Q_{i j}\right]_{\text {off }}=[T]^{T}\left[Q_{i j}\right]_{\text {on }}[T]
$$

in which

$$
\begin{gathered}
{\left[Q_{i j}\right]_{\mathrm{on}}=\left[\begin{array}{ccc}
Q_{11} & Q_{12} & 0 \\
Q_{12} & Q_{22} & 0 \\
0 & 0 & Q_{66}
\end{array}\right],} \\
{[T]=\left[\begin{array}{ccc}
m^{2} & n^{2} & m n \\
n^{2} & m^{2} & -m n \\
-2 m n & 2 m n & m^{2}-n^{2}
\end{array}\right]}
\end{gathered}
$$

with

$$
m=\cos \theta, \quad n=\sin \theta .
$$


The elements of the $\left[Q_{i j}\right]_{\text {on }}$ matrix [13] are

$$
\begin{gathered}
Q_{11}=\left(1-v_{12} \nu_{21}\right)^{-1} E_{11}, \quad Q_{22}=\left(1-v_{12} \nu_{21}\right)^{-1} E_{22}, \\
Q_{12}=\left(1-v_{12} v_{21}\right)^{-1} E_{11} \nu_{21}, \quad Q_{66}=G_{12} .
\end{gathered}
$$

$F_{i}$ and $F_{j}$ of (4) are two shear correction factors presently taken as unity for thin shells and the elements of the $G_{i j}$ matrix are given by

$$
\begin{gathered}
G_{x x}=G_{13} \cos ^{2} \theta+G_{23} \sin ^{2} \theta, \\
G_{x y}=\left(G_{13}-G_{23}\right) \cos \theta \sin \theta, \\
G_{y y}=G_{13} \sin ^{2} \theta+G_{23} \cos ^{2} \theta .
\end{gathered}
$$

The $G_{i j}$ matrix has the form

$$
\left[G_{i j}\right]=\left[\begin{array}{ll}
G_{x x} & G_{x y} \\
G_{x y} & G_{y y}
\end{array}\right]
$$

The strain-displacement relations on the basis of improved first-order approximation theory for thin shell [14] are established as

$$
\begin{aligned}
\left\{\varepsilon_{x}, \varepsilon_{y}, \gamma_{x y}, \gamma_{x z}, \gamma_{y z}\right\}^{T}= & \left\{\varepsilon_{x}^{0}, \varepsilon_{y}^{0}, \gamma_{x y}^{0}, \gamma_{x z}^{0}, \gamma_{y z}^{0}\right\}^{T} \\
& +z\left\{k_{x}, k_{y}, k_{x y}, k_{x z}, k_{y z}\right\}^{T},
\end{aligned}
$$

where, the first vector is the midsurface strain for a hypar shell and the second vector is the curvature. These are given, respectively, by

$$
\left\{\begin{array}{c}
\varepsilon_{x}^{0} \\
\varepsilon_{y}^{0} \\
\gamma_{x y}^{0} \\
\gamma_{x z}^{0} \\
\gamma_{y z}^{0}
\end{array}\right\}=\left\{\begin{array}{c}
\frac{\partial u}{\partial x} \\
\frac{\partial v}{\partial y} \\
\frac{\partial u}{\partial y}+\frac{\partial v}{\partial x}-\frac{2 w}{R_{x y}} \\
\alpha+\frac{\partial w}{\partial x} \\
\beta+\frac{\partial w}{\partial y}
\end{array}\right\}, \quad\left\{\begin{array}{c}
k_{x} \\
k_{y} \\
k_{x y} \\
k_{x z} \\
k_{y z}
\end{array}\right\}=\left\{\begin{array}{c}
\frac{\partial \alpha}{\partial x} \\
\frac{\partial \beta}{\partial y} \\
\frac{\partial \alpha}{\partial y}+\frac{\partial \beta}{\partial x} \\
0 \\
0
\end{array}\right\} .
$$

The radius of cross curvature may be evaluated by differentiating the surface equation of shell in the form $z=f(x, y)$ and for shallow shells which are taken up for the present study the same may be expressed as $1 / R_{x y}=d^{2} z / d x d y$.

2.1. Finite Element Formulation. An eight-noded curved quadratic isoparametric finite element (as shown in Figure 3) is used for hypar shell analysis. The five degrees of freedom taken into consideration at each node are $u, v, w, \alpha, \beta$. The following expressions establish the relations between the displacement at any point with respect to the coordinates $\xi$ and $\eta$ and the nodal degrees of freedom:

$$
\begin{gathered}
u=\sum_{i=1}^{8} N_{i} u_{i}, \quad v=\sum_{i=1}^{8} N_{i} v_{i}, \quad w=\sum_{i=1}^{8} N_{i} w_{i}, \\
\alpha=\sum_{i=1}^{8} N_{i} \alpha_{i}, \quad \beta=\sum_{i=1}^{8} N_{i} \beta_{i},
\end{gathered}
$$

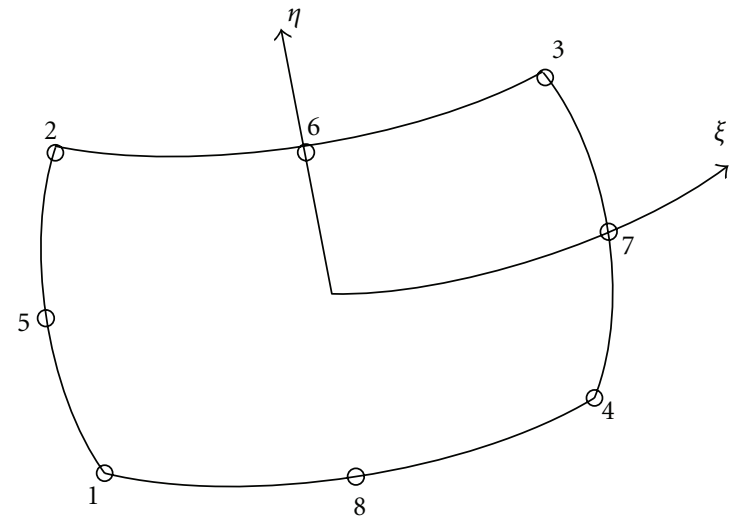

FIGURE 3: Eight noded shell element with isoparametric coordinates.

where the shape functions derived from a cubic interpolation polynomial [14] are

$$
\begin{gathered}
N_{i}=\frac{\left(1+\xi \xi_{i}\right)\left(1+\eta \eta_{i}\right)\left(\xi \xi_{i}+\eta \eta_{i}-1\right)}{4}, \quad \text { for } i=1,2,3,4, \\
N_{i}=\frac{\left(1+\xi \xi_{i}\right)\left(1-\eta^{2}\right)}{2}, \quad \text { for } i=5,7, \\
N_{i}=\frac{\left(1+\eta \eta_{i}\right)\left(1-\xi^{2}\right)}{2}, \quad \text { for } i=6,8 .
\end{gathered}
$$

The generalized displacement vector of an element is expressed in terms of the shape functions and nodal degrees of freedom as

$$
[u]=[N]\left\{d_{e}\right\},
$$

that is,

$$
\{u\}=\left\{\begin{array}{c}
u \\
v \\
w \\
\alpha \\
\beta
\end{array}\right\}=\sum_{i=1}^{8}\left[\begin{array}{lllll}
N_{i} & & & & \\
& N_{i} & & & \\
& & N_{i} & & \\
& & & N_{i} & \\
& & & & N_{i}
\end{array}\right]\left\{\begin{array}{c}
u_{i} \\
v_{i} \\
w_{i} \\
\alpha_{i} \\
\beta_{i}
\end{array}\right\} .
$$

2.2. Element Stiffness Matrix. The strain-displacement relation is given by

$$
\{\varepsilon\}=[B]\left\{d_{e}\right\},
$$

where

$$
[B]=\sum_{i=1}^{8}\left[\begin{array}{ccccc}
N_{i, x} & 0 & 0 & 0 & 0 \\
0 & N_{i, y} & 0 & 0 & 0 \\
N_{i, y} & N_{i, x} & \frac{-2 N_{i}}{R_{x y}} & 0 & 0 \\
0 & 0 & 0 & N_{i, x} & 0 \\
0 & 0 & 0 & 0 & N_{i, y} \\
0 & 0 & 0 & N_{i, y} & N_{i, x} \\
0 & 0 & N_{i, x} & N_{i} & 0 \\
0 & 0 & N_{i, y} & 0 & N_{i}
\end{array}\right] .
$$

The element stiffness matrix is

$$
\left[K_{e}\right]=\iint[B]^{T}[E][B] d x d y .
$$




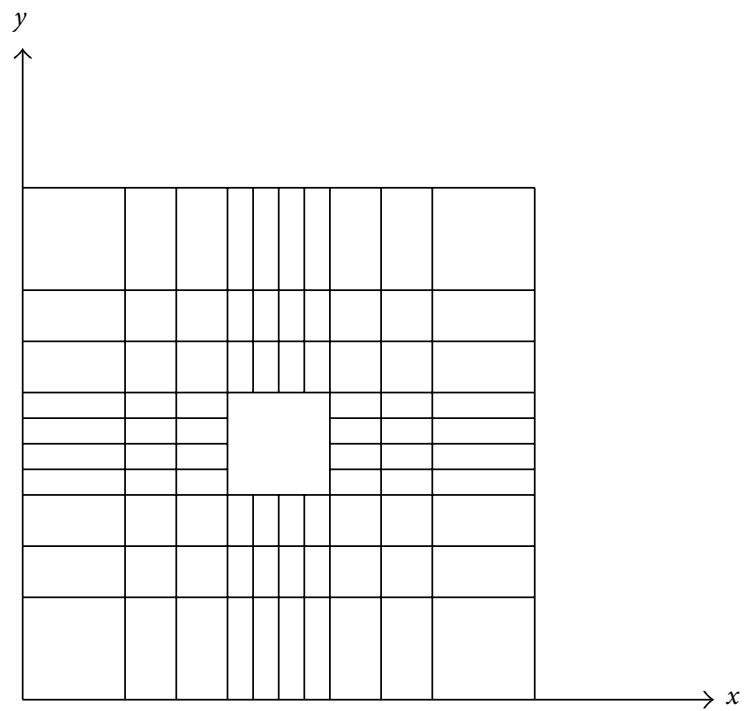

Figure 4: Typical $10 \times 10$ nonuniform mesh arrangements drawn to scale.

2.3. Element Mass Matrix. The element mass matrix is obtained from the integral

$$
\left[M_{e}\right]=\iint[N]^{T}[P][N] d x d y
$$

where

$$
\begin{gathered}
{[N]=\sum_{i=1}^{8}\left[\begin{array}{ccccc}
N_{i} & 0 & 0 & 0 & 0 \\
0 & N_{i} & 0 & 0 & 0 \\
0 & 0 & N_{i} & 0 & 0 \\
0 & 0 & 0 & N_{i} & 0 \\
0 & 0 & 0 & 0 & N_{i}
\end{array}\right],} \\
{[P]=\sum_{i=1}^{8}\left[\begin{array}{lllll}
P & 0 & 0 & 0 & 0 \\
0 & P & 0 & 0 & 0 \\
0 & 0 & P & 0 & 0 \\
0 & 0 & 0 & I & 0 \\
0 & 0 & 0 & 0 & I
\end{array}\right],}
\end{gathered}
$$

in which

$$
P=\sum_{k=1}^{\mathrm{np}} \int_{z_{k-1}}^{z_{k}} \rho d z, \quad I=\sum_{k=1}^{\mathrm{np}} \int_{z_{k-1}}^{z_{k}} z \rho d z .
$$

2.4. Modeling the Cutout. The code developed can take the position and size of cutout as input. The program is capable of generating nonuniform finite element mesh all over the shell surface. So the element size is gradually decreased near the cutout margins. One such typical mesh arrangement is shown in Figure 4. Such finite element mesh is redefined in steps, and a particular grid is chosen to obtain the fundamental frequency when the result does not improve by more than one percent on further refining. Convergence of results is ensured in all the problems taken up here.

2.5. Solution Procedure for Dynamic Analysis. The element stiffness and mass matrices are evaluated first by expressing

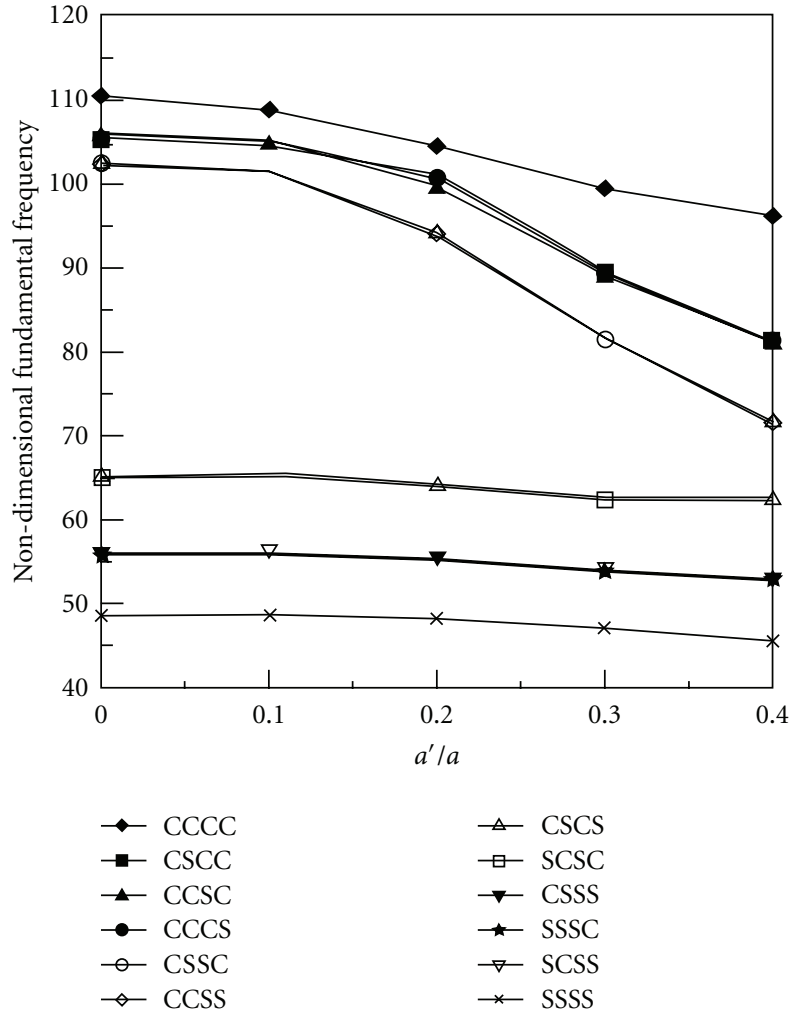

FIGURE 5: Values of non-dimensional fundamental frequency $(\bar{\omega})$ of 0/90/0/90 hypar shell with cutout for different sizes of central cutout and boundary conditions. $a / b=1, a / h=100, a^{\prime} / a=0.1, a^{\prime} / b^{\prime}=1$, $c / a=0.2 ; E_{11} / E_{22}=25, G_{23}=0.2 E_{22}, G_{13}=G_{12}=0.5 E_{22}, v_{12}=$ $\nu_{21}=0.25$.

the integrals in the local natural coordinates $\xi$ and $\eta$ of the $2 \times 2$ Gauss quadrature because the shape functions are derived from a cubic interpolation polynomial (Dey et al. [14]) and it is an established fact that a polynomial of degree $2 n-1$ is integrated exactly by $n$ point Gauss quadrature [15]. Then the element matrices are assembled after performing appropriate transformations due to the curved shell surface to obtain the respective global matrices $[K]$ and $[M]$.

The free vibration analysis involves determination of natural frequencies from the condition

$$
\left|[K]-\omega^{2}[M]\right|=0 .
$$

This is a generalized eigen value problem and is solved by the subspace iteration algorithm.

\section{Validation Study}

The accuracy of the present formulation is first validated by comparing the results of the following problem available in the existing literature.

Free vibration of simply supported and clamped hypar shell with $(0 / 90)_{4}$ shell of aspect ratio $a / b=1, c / a=0.2$, and side to thickness ratio $a / h=100$ with cutouts [1] is considered as the benchmark problem. The nondimensional frequency parameter is $\bar{\omega}=\omega a^{2}\left(\rho / E_{22} h^{2}\right)^{1 / 2}$. Material 
TABLE 1: Non-dimensional fundamental frequencies $(\bar{\omega})$ for hypar shells (lamination $\left.(0 / 90)_{4}\right)$ with concentric cutouts.

\begin{tabular}{|c|c|c|c|c|c|c|c|c|}
\hline \multirow{3}{*}{$a^{\prime} / a$} & \multicolumn{2}{|c|}{ Chakravorty et al. [1] } & \multicolumn{6}{|c|}{ Present finite element model } \\
\hline & \multirow{2}{*}{ Simply supported } & \multirow{2}{*}{ Clamped } & \multicolumn{3}{|c|}{ Simply supported } & \multicolumn{3}{|c|}{ Clamped } \\
\hline & & & $8 \times 8$ & $10 \times 10$ & $12 \times 12$ & $8 \times 8$ & $10 \times 10$ & $12 \times 12$ \\
\hline 0.0 & 50.829 & 111.600 & 50.573 & 50.821 & 50.825 & 111.445 & 111.592 & 111.612 \\
\hline 0.1 & 50.769 & 110.166 & 50.679 & 50.758 & 50.779 & 109.987 & 110.057 & 110.233 \\
\hline 0.2 & 50.434 & 105.464 & 50.323 & 50.421 & 50.400 & 105.265 & 105.444 & 105.443 \\
\hline 0.3 & 49.165 & 101.350 & 49.045 & 49.157 & 49.178 & 101.110 & 101.340 & 101.490 \\
\hline 0.4 & 47.244 & 97.987 & 47.132 & 47.242 & 47.141 & 97.670 & 97.985 & 97.991 \\
\hline
\end{tabular}

$a / b=1, a / h=100, a^{\prime} / b^{\prime}=1, c / a=0.2 ; E_{11} / E_{22}=25, G_{23}=0.2 E_{22}, G_{13}=G_{12}=0.5 E_{22}, \nu_{12}=v_{21}=0.25$.

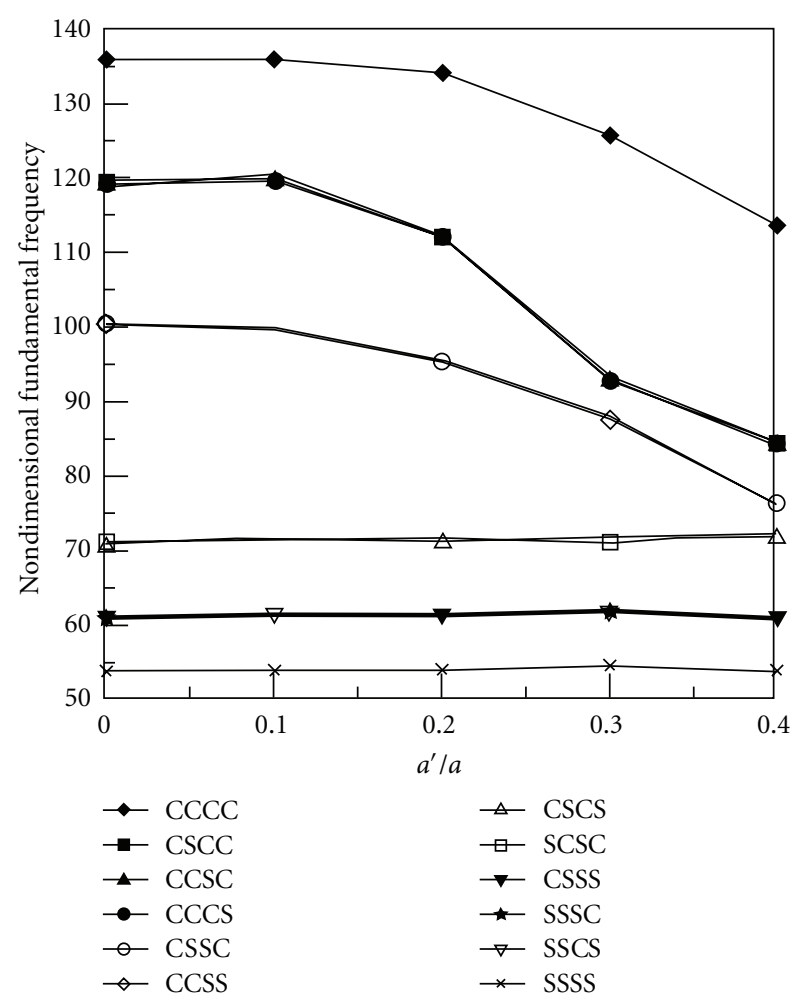

Figure 6: Values of non-dimensional fundamental frequency $(\bar{\omega})$ of $+45 /-45 /+45 /-45$ hypar shell with cutout for different sizes of central cutout and boundary conditions. $a / b=1, a / h=100, a^{\prime} / a=$ $0.1, a^{\prime} / b^{\prime}=1, c / a=0.2 ; E_{11} / E_{22}=25, G_{23}=0.2 E_{22}, G_{13}=G_{12}=$ $0.5 E_{22}, v_{12}=v_{21}=0.25$.

properties are as follows: $E_{11} / E_{22}=25, G_{23}=0.2 E_{22}, G_{13}=$ $G_{12}=0.5 E_{22}, v_{12}=v_{21}=0.25$.

The fundamental frequencies of hypar shell with cutout obtained by the present method agree well with those reported by Chakravorty et al. [1] as evident from Table 1, establishing the correctness of the present results. The fact that the cutouts are properly modeled in the present formulation is thus also established. The present approach uses the improved first-order approximation theory for thin shells [13] considering the radius of cross curvature. For this class of thin shells a shear correction factor of unity is found to yield good results. It is observed that the results remain the same when analysis is repeated with the commonly used shear correction factor of $\pi / \sqrt{12}$.

\section{Results and Discussion}

In order to study the effect of cutout size on the free vibration response additional problems for hypar shells with 0/90/0/90 and +45/-45/+45/-45 lamination and different boundary conditions have been solved. The selection of the $0 / 90 / 0 / 90$ and $+45 /-45 /+45 /-45$ lamination is based on an earlier study by Sahoo and Chakravorty [16] which revealed that repeating 0/90 unit and $+45 /-45$ unit more than once and keeping the total shell thickness constant do not improve the fundamental frequency to an appreciable extent. The positions of the cutouts are varied along both of the plan directions of the shell for different practical boundary conditions to study the effect of eccentricity of cutout on the fundamental frequency.

4.1. Free Vibration Behaviour of Shells with Concentric Cutouts. Figures 5 and 6 furnish the results of non-dimensional frequency $(\bar{\omega})$ of $0 / 90 / 0 / 90$ and $+45 /-45 /+45 /-45$ hypar shells. The shells considered are of square plan form $(a=b)$, and the cutouts are also taken to be square in plan $\left(a^{\prime}=b^{\prime}\right)$. The cutouts are placed concentrically on the shell surface. The cutout sizes (i.e., $a^{\prime} / a$ ) are varied from 0 to 0.4 , and boundary conditions are varied along the four edges. The boundary conditions are designated by describing the support clamped or simply supported as $\mathrm{C}$ or $\mathrm{S}$ taken in an anticlockwise order from the edge $x=0$. This means a shell with CSCS boundary is clamped along $x=0$, simply supported along $y=0$ and clamped along $x=a$ and simply supported along $y=b$. The material and geometric properties of shells and cutouts are mentioned along with the figures.

4.1.1. Effect of Cutout Sizes. From the figures it is seen that when a cutout is introduced to a shell the fundamental frequency increases in 6 out of 12 cases in case of symmetric crossply shell. But in case of symmetric angle ply one the fundamental frequency increases in 10 out of 12 cases. In order to study the effect of cutout size, in more detail, the ratio of the fundamental frequency of a concentric punctured shell to that of a shell without cutout is expressed in percentage. The increase or decrease in percentage of fundamental frequency from the full shell is denoted by p. Tables 2 and 3 contain such $p$ values for $0 / 90 / 0 / 90$ and $+45 /-45 /+45 /-45$ shells, respectively. Negative sign 
TABLe 2: Values of “p” for 0/90/0/90 hypar shell.

\begin{tabular}{|c|c|c|c|c|c|}
\hline \multirow{2}{*}{ Boundary conditions } & \multicolumn{5}{|c|}{ Cutout size $\left(a^{\prime} / a\right)$} \\
\hline & 0 & 0.1 & 0.2 & 0.3 & 0.4 \\
\hline CCCC & 0 & -1.56 & -5.48 & -10.01 & -12.94 \\
\hline CSCC & 0 & -0.9 & -3.87 & -14.97 & -22.62 \\
\hline $\operatorname{CCSC}$ & 0 & -0.99 & -5.88 & -16.04 & -23.3 \\
\hline CCCS & 0 & -0.9 & -4.33 & -15.42 & -22.76 \\
\hline CSSC & 0 & -1.12 & -8.24 & -20.47 & -30.27 \\
\hline CCSS & 0 & -1.12 & -8.24 & -20.47 & -30.27 \\
\hline CSCS & 0 & 0.38 & -1.67 & -4.16 & -4.21 \\
\hline SCSC & 0 & 0.38 & -1.71 & -4.29 & -4.43 \\
\hline CSSS & 0 & 0.3 & -1.05 & -3.35 & -5.53 \\
\hline SSSC & 0 & 0.3 & -1.09 & -3.45 & -5.74 \\
\hline SSCS & 0 & 0.33 & -1.06 & -3.35 & -5.54 \\
\hline SSSS & 0 & 0.23 & -0.63 & -2.92 & -6.08 \\
\hline
\end{tabular}

$a / b=1, a / h=100, a^{\prime} / b^{\prime}=1, c / a=0.2 ; E_{11} / E_{22}=25, G_{23}=0.2 E_{22}, G_{13}=G_{12}=0.5 E_{22}, v_{12}=v_{21}=0.25$.

TABLe 3: Values of " $p$ ” for $+45 /-45 /+45 /-45$ hypar shell.

\begin{tabular}{|c|c|c|c|c|c|}
\hline \multirow{2}{*}{ Boundary conditions } & \multicolumn{5}{|c|}{ Cutout size $\left(a^{\prime} / a\right)$} \\
\hline & 0 & 0.1 & 0.2 & 0.3 & 0.4 \\
\hline 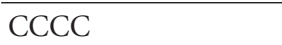 & 0 & 0.08 & -1.2 & -7.4 & -16.29 \\
\hline CsCC & 0 & 1.13 & -6.11 & -21.59 & -29.25 \\
\hline CCSC & 0 & 0.67 & -5.84 & -21.9 & -28.99 \\
\hline CCCS & 0 & 0.75 & -5.78 & -21.96 & -29.22 \\
\hline CSSC & 0 & -0.82 & -5.14 & -12.44 & -24.09 \\
\hline CCSS & 0 & -0.97 & -5.18 & -12.77 & -24.19 \\
\hline CSCS & 0 & 0.99 & 0.53 & 1.53 & 1.52 \\
\hline SCSC & 0 & 0.93 & 0.53 & -0.01 & 1.87 \\
\hline CSSS & 0 & 0.77 & 0.64 & 1.36 & -0.29 \\
\hline SSSC & 0 & 0.74 & 0.64 & 1.34 & -0.07 \\
\hline SSCS & 0 & 0.58 & 0.64 & 1.35 & -0.3 \\
\hline SSSS & 0 & 0.44 & 0.8 & 1.66 & 0.36 \\
\hline
\end{tabular}

$a / b=1, a / h=100, a^{\prime} / b^{\prime}=1, c / a=0.2 ; E_{11} / E_{22}=25, G_{23}=0.2 E_{22}, G_{13}=G_{12}=0.5 E_{22}, v_{12}=v_{21}=0.25$.

indicates decrease in frequency. It is evident from Tables 2 and 3 that in all the cases with the introduction of cutout with $a^{\prime} / a=0.1$ the increase or decrease in frequency is not more than $1 \%$. But with further increase in cutout size, that is, when $a^{\prime} / a=0.2$, fundamental frequency decreases in all the cases, except for simply supported symmetric angle ply shells. Because in such cases loss of stiffness is more significant than loss of mass. Here also the decrease in fundamental frequency is between 1 and $10 \%$. But with further increase in cutout sizes $a^{\prime} / a=0.3$ and 0.4 fundamental frequency decreases to an appreciable extent (up to $30 \%$ ). For some cases of symmetric angle ply shell no such unified trend is observed. This leads to the engineering conclusion that concentric cutouts may be provided safely on shell surfaces for functional requirements up to $a^{\prime} / a=0.2$.

4.1.2. Effect of Boundary Conditions. The boundary conditions have been divided into four groups, so that the combinations in a particular group have equal number of boundary reactions. The groups are of the following forms:

Group I: contains CCCC shells.

Group II: contains CSCC, CCSC, and CCCS shells.

Group III: contains CSSC, CCSS, CSCS, and SCSC shells.

Group IV: contains SSSS shells.

As evident from Figures 5 and 6, fundamental frequencies of members belonging to different boundary combinations may be grouped according to performance.

According to the values of $(\bar{\omega})$, the group III may be subdivided into Group IIIa and Group IIIb for both 0/90/0/90 and $+45 /-45 /+45 /-45$ shells.

Group I: contains CCCC shells.

Group II: contains CSCC, CCSC, and CCCS shells. 
TABLE 4: Clamping options for 0/90/0/90 hypar shells with central cutouts having $a^{\prime} / a$ ratio 0.2 .

\begin{tabular}{|c|c|c|c|}
\hline $\begin{array}{l}\text { Number of sides to } \\
\text { be clamped }\end{array}$ & Clamped edges & $\begin{array}{l}\text { Improvement of frequencies with } \\
\text { respect to simply supported shells }\end{array}$ & $\begin{array}{l}\text { Marks indicating the } \\
\text { efficiencies of clamping }\end{array}$ \\
\hline 0 & $\begin{array}{l}\text { Simply supported no edges } \\
\text { clamped (SSSS) }\end{array}$ & - & 0 \\
\hline \multirow{3}{*}{1} & (a) Along $x=0$ (CSSS) & Slight improvement & 13 \\
\hline & (b) along $x=a$ (SSCS) & Slight improvement & 13 \\
\hline & (c) along $y=b$ (SSSC) & Slight improvement & 13 \\
\hline \multirow[t]{2}{*}{2} & $\begin{array}{l}\text { (a) Two alternate edges } \\
\text { (CSCS, SCSC) }\end{array}$ & Good improvement & 28 \\
\hline & $\begin{array}{l}\text { (b) Two adjacent edges } \\
\text { (CSSC, CCSS) }\end{array}$ & Marked improvement & 82 \\
\hline \multirow{3}{*}{3} & $\begin{array}{l}3 \text { edges excluding } y=0 \\
\text { CSCC }\end{array}$ & Remarkable improvement and & 94 \\
\hline & $\begin{array}{l}3 \text { edges excluding } x=a \\
\text { CCSC }\end{array}$ & $\begin{array}{l}\text { frequency becomes almost equal to } \\
\text { that of fully clamped shells }\end{array}$ & 92 \\
\hline & $\begin{array}{l}3 \text { edges excluding } y=b \\
\text { CCCS }\end{array}$ & & 93 \\
\hline 4 & All sides (CCCC) & Frequency attains a maximum value & 100 \\
\hline
\end{tabular}

TABLE 5: Clamping options for $+45 /-45 /+45 /-45$ hypar shells with central cutouts having a $/$ a ratio 0.2 .

\begin{tabular}{llll}
\hline $\begin{array}{l}\text { Number of sides } \\
\text { to be clamped }\end{array}$ & Clamped edges & $\begin{array}{l}\text { Improvement of frequencies with } \\
\text { respect to simply supported shells }\end{array}$ & $\begin{array}{c}\text { Marks indicating the } \\
\text { efficiencies of clamping }\end{array}$ \\
\hline 0 & $\begin{array}{l}\text { Simply supported no edges clamped } \\
\text { (SSSS) }\end{array}$ & Slight improvement & 0 \\
& (a) Along $x=0$ (CSSS) & Slight improvement & 9 \\
1 & (b) along $x=a$ (SSCS) & Slight improvement & 9 \\
& (c) along $y=b$ (SSSC) & Good improvement & 22 \\
2 & (a) Two alternate edges (CSCS, SCSC) & Marked improvement & 52 \\
& (b) Two adjacent edges (CSSC, CCSS) & Remarkable improvement & 72 \\
3 & 3 edges excluding $y=0$ CSCC & Remarkable improvement & 72 \\
4 & 3 edges excluding $x=a$ CCSC & Remarkable improvement & 72 \\
& 3 edges excluding $y=b$ CCCS & Frequency attains a maximum value & 100 \\
\hline
\end{tabular}

Group IIIa: contains CSSC, CCSS shells.

Group IIIb: contains CSCS and SCSC shells.

Group IV: contains SSSS shells.

This observation indicates that the impact of arrangement of boundary constraints is far more important than their actual number in determining the free vibration characteristics.

The frequencies are further studied, and marks are given to the options of clamping the edges of a simply supported shell in order to gradually improve performances. Tables 4 and 5 furnish such clamping options for crossply and angle ply shells, respectively. The scale is chosen like this: 0 is assigned to a simply supported shell and 100 to a clamped shell. These marks are furnished for cutouts with $a^{\prime} / a=0.2$. These tables will help a practicing engineer. If one takes the frequency of a clamped shell as upper limit and that of the simply supported as lower limit, one can easily realize the efficiency of a particular boundary condition.
4.2. Effect of Eccentricity of Cutout Position on Fundamental Frequency. To study the effect of cutout positions on fundamental frequencies, results are obtained for different locations of a cutout with $a^{\prime} / a=0.2$. As with the introduction of cutout with $a^{\prime} / a=0.2$, the change in fundamental frequency with that of an unpunctured shell is within $1-10 \%$, so $a^{\prime} / a=0.2$ is chosen for the further study. Each of the non-dimensional coordinates of the cutout centre $(\bar{x}=x / a, \bar{y}=y / a)$ is varied from 0.2 to 0.8 along both the plan directions so that the distance of a cutout margin from the shell boundary is not less than one-tenth of the plan dimension of the shell. The study is carried out for all the twelve boundary conditions for both $0 / 90 / 0 / 90$ and $+45 /-45 /+45 /-45$ hypar shells. The ratio of the fundamental frequency of a shell with an eccentric puncture to that of a shell with concentric puncture (obtainable from Figures 5 and 6) expressed in percentage is denoted by $r$. Tables 6 and 7 contain the value of $r$ for $0 / 90 / 0 / 90$ and $+45 /-45 /+45 /-45$ hypar shells. 
TABLE 6: Values of " $r$ " for 0/90/0/90 hypar shells.

\begin{tabular}{|c|c|c|c|c|c|c|c|c|}
\hline \multirow{2}{*}{ Edge condition } & \multirow{2}{*}{$\bar{y}$} & \multicolumn{7}{|c|}{$\bar{x}$} \\
\hline & & 0.2 & 0.3 & 0.4 & 0.5 & 0.6 & 0.7 & 0.8 \\
\hline \multirow{7}{*}{ CCCC } & 0.2 & 98.34 & 98.04 & 97.82 & 97.7 & 97.77 & 97.98 & 98.29 \\
\hline & 0.3 & 98.13 & 97.49 & 96.74 & 96.4 & 96.71 & 97.46 & 98.1 \\
\hline & 0.4 & 98.02 & 96.88 & 95.55 & 95.04 & 95.54 & 96.88 & 98.02 \\
\hline & 0.5 & 97.98 & 96.62 & 95.08 & 94.52 & 95.08 & 96.62 & 97.98 \\
\hline & 0.6 & 98.02 & 96.88 & 95.54 & 95.04 & 95.55 & 96.88 & 98.02 \\
\hline & 0.7 & 98.1 & 97.46 & 96.71 & 96.4 & 96.74 & 97.49 & 98.13 \\
\hline & 0.8 & 98.29 & 97.98 & 97.77 & 97.71 & 97.82 & 98.04 & 98.34 \\
\hline \multirow{7}{*}{ CSCC } & 0.2 & 96.11 & 96.68 & 96.37 & 96.31 & 96.38 & 96.67 & 97.25 \\
\hline & 0.3 & 96.11 & 95.46 & 95.31 & 95.45 & 95.38 & 95.50 & 96.1 \\
\hline & 0.4 & 95.35 & 94.72 & 94.81 & 95.51 & 94.88 & 94.75 & 95.36 \\
\hline & 0.5 & 95.27 & 94.51 & 94.49 & 96.13 & 94.49 & 94.52 & 95.27 \\
\hline & 0.6 & 95.49 & 94.58 & 94.05 & 93.43 & 94.03 & 94.59 & 95.51 \\
\hline & 0.7 & 95.83 & 94.81 & 93.71 & 92.57 & 93.68 & 94.83 & 95.87 \\
\hline & 0.8 & 102.93 & 95.19 & 93.75 & 92.41 & 93.74 & 95.22 & 96.33 \\
\hline \multirow{7}{*}{ CCSC } & 0.2 & 96.19 & 95.74 & 95.42 & 95.26 & 95.36 & 95.95 & 97.05 \\
\hline & 0.3 & 95.08 & 94.73 & 94.51 & 94.50 & 94.77 & 95.49 & 96.76 \\
\hline & 0.4 & 93.26 & 93.26 & 93.65 & 94.21 & 94.77 & 95.44 & 96.73 \\
\hline & 0.5 & 91.67 & 91.87 & 92.74 & 94.12 & 95.51 & 95.61 & 96.81 \\
\hline & 0.6 & 93.21 & 93.21 & 93.58 & 94.15 & 94.77 & 95.48 & 96.76 \\
\hline & 0.7 & 95.05 & 94.69 & 94.47 & 94.48 & 94.80 & 95.57 & 96.84 \\
\hline & 0.8 & 96.19 & 95.73 & 95.41 & 95.28 & 95.42 & 96.08 & 97.17 \\
\hline \multirow{7}{*}{ CCCS } & 0.2 & 96.32 & 95.22 & 93.73 & 92.33 & 93.65 & 95.16 & 95.16 \\
\hline & 0.3 & 95.86 & 94.83 & 93.65 & 92.44 & 93.59 & 94.78 & 95.83 \\
\hline & 0.4 & 95.50 & 94.59 & 94.02 & 93.38 & 93.97 & 94.56 & 95.48 \\
\hline & 0.5 & 95.28 & 94.52 & 94.47 & 95.68 & 94.43 & 94.49 & 95.26 \\
\hline & 0.6 & 95.34 & 94.49 & 94.85 & 95.51 & 94.79 & 94.71 & 95.33 \\
\hline & 0.7 & 96.08 & 95.50 & 95.37 & 95.45 & 95.31 & 95.46 & 96.09 \\
\hline & 0.8 & 97.23 & 96.67 & 96.38 & 96.31 & 96.37 & 96.68 & 97.28 \\
\hline \multirow{7}{*}{ CSSC } & 0.2 & 96.08 & 95.31 & 94.74 & 94.43 & 94.54 & 95.05 & 95.64 \\
\hline & 0.3 & 95.38 & 95.13 & 95.25 & 95.43 & 95.50 & 95.44 & 95.10 \\
\hline & 0.4 & 91.75 & 91.74 & 92.23 & 93.10 & 94.94 & 95.44 & 94.57 \\
\hline & 0.5 & 90.85 & 90.57 & 90.76 & 91.46 & 93.56 & 95.23 & 94.56 \\
\hline & 0.6 & 92.37 & 91.62 & 91.32 & 91.40 & 92.88 & 94.92 & 94.98 \\
\hline & 0.7 & 94.10 & 93.04 & 92.27 & 91.43 & 92.24 & 94.84 & 95.64 \\
\hline & 0.8 & 95.61 & 94.49 & 93.33 & 91.74 & 92.13 & 95.18 & 96.46 \\
\hline \multirow{7}{*}{ CCSS } & 0.2 & 95.54 & 94.41 & 93.26 & 91.63 & 91.98 & 94.95 & 96.31 \\
\hline & 0.3 & 94.05 & 92.98 & 92.21 & 91.34 & 92.10 & 94.66 & 95.50 \\
\hline & 0.4 & 92.36 & 91.61 & 91.32 & 91.34 & 92.80 & 94.77 & 94.87 \\
\hline & 0.5 & 90.91 & 90.66 & 90.80 & 91.76 & 93.68 & 95.11 & 94.50 \\
\hline & 0.6 & 91.71 & 91.75 & 92.27 & 93.17 & 95.24 & 95.40 & 94.55 \\
\hline & 0.7 & 95.28 & 95.03 & 95.14 & 95.36 & 95.47 & 95.43 & 95.11 \\
\hline & 0.8 & 95.96 & 95.22 & 94.69 & 94.44 & 94.62 & 95.18 & 95.77 \\
\hline \multirow{7}{*}{ CSCS } & 0.2 & 99.07 & 100.14 & 98.96 & 98.08 & 98.93 & 100.14 & 99.10 \\
\hline & 0.3 & 99.27 & 100.79 & 99.18 & 97.98 & 99.14 & 100.76 & 99.28 \\
\hline & 0.4 & 99.56 & 101.15 & 99.46 & 98.20 & 99.44 & 101.13 & 99.55 \\
\hline & 0.5 & 99.67 & 101.24 & 99.57 & 98.33 & 99.56 & 101.24 & 99.67 \\
\hline & 0.6 & 99.54 & 101.13 & 99.44 & 98.20 & 99.45 & 101.15 & 99.55 \\
\hline & 0.7 & 99.28 & 100.76 & 99.14 & 97.98 & 99.17 & 100.78 & 99.27 \\
\hline & 0.8 & 99.10 & 100.14 & 98.92 & 98.08 & 98.95 & 100.14 & 99.07 \\
\hline
\end{tabular}


Table 6: Continued.

\begin{tabular}{|c|c|c|c|c|c|c|c|c|}
\hline \multirow{2}{*}{ Edge condition } & \multirow{2}{*}{$\bar{y}$} & \multicolumn{7}{|c|}{$\bar{x}$} \\
\hline & & 0.2 & 0.3 & 0.4 & 0.5 & 0.6 & 0.7 & 0.8 \\
\hline \multirow{7}{*}{ SCSC } & 0.2 & 99.04 & 99.30 & 99.56 & 99.67 & 99.56 & 99.27 & 98.99 \\
\hline & 0.3 & 100.18 & 100.79 & 101.13 & 101.23 & 101.14 & 100.81 & 100.18 \\
\hline & 0.4 & 99.04 & 99.22 & 99.43 & 99.54 & 99.44 & 99.23 & 99.04 \\
\hline & 0.5 & 98.17 & 98.07 & 98.20 & 98.29 & 98.20 & 98.07 & 98.17 \\
\hline & 0.6 & 99.04 & 99.23 & 99.44 & 99.54 & 99.43 & 99.22 & 99.04 \\
\hline & 0.7 & 100.18 & 100.82 & 101.14 & 101.23 & 101.13 & 100.80 & 100.18 \\
\hline & 0.8 & 98.99 & 99.27 & 99.56 & 99.82 & 99.56 & 99.30 & 99.04 \\
\hline \multirow{7}{*}{ CSSS } & 0.2 & 98.49 & 98.97 & 98.19 & 97.42 & 97.62 & 98.61 & 98.94 \\
\hline & 0.3 & 98.82 & 99.92 & 99.27 & 98.39 & 98.59 & 99.53 & 99.48 \\
\hline & 0.4 & 99.28 & 100.60 & 99.91 & 98.83 & 98.95 & 99.95 & 99.89 \\
\hline & 0.5 & 99.44 & 100.78 & 100.07 & 98.95 & 99.07 & 100.12 & 100.07 \\
\hline & 0.6 & 99.26 & 100.59 & 99.89 & 98.82 & 98.97 & 99.98 & 99.89 \\
\hline & 0.7 & 98.83 & 99.91 & 99.22 & 98.37 & 98.62 & 99.58 & 99.49 \\
\hline & 0.8 & 98.54 & 99.02 & 98.17 & 97.43 & 97.67 & 98.65 & 98.93 \\
\hline \multirow{7}{*}{ SSSC } & 0.2 & 98.92 & 99.40 & 99.86 & 100.08 & 99.87 & 99.41 & 98.90 \\
\hline & 0.3 & 98.71 & 99.58 & 99.94 & 100.10 & 99.95 & 99.59 & 98.70 \\
\hline & 0.4 & 97.69 & 98.68 & 98.96 & 99.05 & 98.96 & 98.67 & 97.67 \\
\hline & 0.5 & 97.44 & 98.46 & 98.82 & 98.91 & 98.82 & 98.44 & 97.42 \\
\hline & 0.6 & 98.23 & 99.29 & 99.86 & 100.01 & 99.85 & 99.27 & 98.22 \\
\hline & 0.7 & 99.00 & 99.94 & 100.56 & 100.74 & 100.55 & 99.93 & 99.01 \\
\hline & 0.8 & 98.39 & 98.81 & 99.26 & 99.43 & 99.26 & 98.83 & 98.45 \\
\hline \multirow{7}{*}{ SSCS } & 0.2 & 98.93 & 98.65 & 97.68 & 97.42 & 98.18 & 99.02 & 98.55 \\
\hline & 0.3 & 99.48 & 99.58 & 98.62 & 98.37 & 99.22 & 99.91 & 98.83 \\
\hline & 0.4 & 99.89 & 99.98 & 98.96 & 98.82 & 99.88 & 100.59 & 99.27 \\
\hline & 0.5 & 100.08 & 100.12 & 99.06 & 98.94 & 100.06 & 100.78 & 99.45 \\
\hline & 0.6 & 99.88 & 99.95 & 98.94 & 98.82 & 99.90 & 100.60 & 99.27 \\
\hline & 0.7 & 99.48 & 99.53 & 98.58 & 98.39 & 99.26 & 99.91 & 98.81 \\
\hline & 0.8 & 98.94 & 98.60 & 97.61 & 97.42 & 98.17 & 98.96 & 98.49 \\
\hline \multirow{7}{*}{ SSSS } & 0.2 & 97.78 & 97.55 & 96.93 & 96.64 & 96.85 & 97.51 & 97.94 \\
\hline & 0.3 & 98.22 & 99.14 & 98.94 & 98.81 & 98.80 & 98.99 & 98.06 \\
\hline & 0.4 & 97.52 & 99.41 & 99.42 & 99.28 & 99.24 & 99.20 & 97.43 \\
\hline & 0.5 & 97.22 & 99.41 & 99.58 & 99.37 & 99.59 & 99.41 & 97.22 \\
\hline & 0.6 & 97.44 & 99.20 & 99.23 & 99.27 & 99.43 & 99.41 & 97.52 \\
\hline & 0.7 & 98.06 & 98.99 & 98.80 & 98.81 & 98.93 & 99.14 & 98.22 \\
\hline & 0.8 & 97.94 & 97.50 & 96.84 & 96.66 & 96.93 & 97.55 & 97.78 \\
\hline
\end{tabular}

$a / b=1, a / h=100, a^{\prime} / b^{\prime}=1, c / a=0.2 ; E_{11} / E_{22}=25, G_{23}=0.2 E_{22}, G_{13}=G_{12}=0.5 E_{22}, v_{12}=v_{21}=0.25$. 
TABLE 7: Values of " $r$ " for $+45 /-45 /+45 /-45$ hypar shells.

\begin{tabular}{|c|c|c|c|c|c|c|c|c|}
\hline \multirow{2}{*}{ Edge condition } & \multirow{2}{*}{$\bar{y}$} & \multicolumn{7}{|c|}{$\bar{x}$} \\
\hline & & 0.2 & 0.3 & 0.4 & 0.5 & 0.6 & 0.7 & 0.8 \\
\hline \multirow{7}{*}{ CCCC } & 0.2 & 97.79 & 94.67 & 95.97 & 102.56 & 95.97 & 94.82 & 97.78 \\
\hline & 0.3 & 94.87 & 94.55 & 96.55 & 101.00 & 96.53 & 94.55 & 94.87 \\
\hline & 0.4 & 95.96 & 96.43 & 97.07 & 98.73 & 97.07 & 96.43 & 95.95 \\
\hline & 0.5 & 102.07 & 100.76 & 98.61 & 98.80 & 98.61 & 100.76 & 101.92 \\
\hline & 0.6 & 95.95 & 96.43 & 97.10 & 98.77 & 97.07 & 96.43 & 95.95 \\
\hline & 0.7 & 94.87 & 94.55 & 96.52 & 100.99 & 96.52 & 94.55 & 94.87 \\
\hline & 0.8 & 97.79 & 94.82 & 95.97 & 102.30 & 95.97 & 94.82 & 97.78 \\
\hline \multirow{7}{*}{ CSCC } & 0.2 & 97.57 & 94.48 & 97.00 & 104.29 & 97.32 & 94.75 & 97.73 \\
\hline & 0.3 & 99.55 & 97.12 & 98.28 & 101.72 & 98.39 & 97.16 & 99.54 \\
\hline & 0.4 & 100.05 & 97.96 & 97.40 & 99.02 & 97.28 & 97.79 & 101.05 \\
\hline & 0.5 & 100.24 & 97.51 & 94.53 & 93.90 & 94.31 & 97.28 & 100.18 \\
\hline & 0.6 & 97.85 & 95.01 & 91.14 & 90.09 & 90.91 & 94.74 & 97.89 \\
\hline & 0.7 & 97.55 & 92.19 & 87.69 & 86.65 & 87.45 & 91.90 & 97.32 \\
\hline & 0.8 & 97.21 & 90.03 & 85.03 & 84.15 & 84.80 & 89.75 & 97.08 \\
\hline \multirow{7}{*}{ CCSC } & 0.2 & 97.20 & 97.40 & 98.19 & 100.24 & 99.74 & 99.45 & 97.77 \\
\hline & 0.3 & 89.82 & 91.97 & 94.82 & 97.28 & 97.77 & 97.17 & 94.74 \\
\hline & 0.4 & 84.74 & 87.48 & 90.99 & 94.48 & 97.44 & 98.49 & 97.24 \\
\hline & 0.5 & 84.02 & 86.63 & 90.17 & 94.16 & 99.51 & 102.03 & 104.53 \\
\hline & 0.6 & 84.74 & 87.47 & 90.99 & 94.48 & 97.44 & 98.49 & 97.24 \\
\hline & 0.7 & 89.82 & 91.97 & 94.82 & 97.28 & 97.77 & 97.17 & 94.74 \\
\hline & 0.8 & 97.20 & 97.40 & 98.19 & 100.24 & 99.74 & 99.48 & 97.77 \\
\hline \multirow{7}{*}{ CCCS } & 0.2 & 97.12 & 89.92 & 85.08 & 84.42 & 85.08 & 89.92 & 97.12 \\
\hline & 0.3 & 97.36 & 92.03 & 87.73 & 86.93 & 87.73 & 92.03 & 97.36 \\
\hline & 0.4 & 98.00 & 94.81 & 91.17 & 90.40 & 91.17 & 94.81 & 98.00 \\
\hline & 0.5 & 100.15 & 97.25 & 90.40 & 94.22 & 94.51 & 97.25 & 100.15 \\
\hline & 0.6 & 99.96 & 97.83 & 97.38 & 99.37 & 97.38 & 97.83 & 100.67 \\
\hline & 0.7 & 99.58 & 97.23 & 98.44 & 101.84 & 98.44 & 97.23 & 99.95 \\
\hline & 0.8 & 97.74 & 94.71 & 97.22 & 104.30 & 97.22 & 94.71 & 97.76 \\
\hline \multirow{7}{*}{ CSSC } & 0.2 & 98.26 & 99.11 & 101.00 & 99.30 & 96.94 & 95.60 & 95.03 \\
\hline & 0.3 & 90.66 & 92.23 & 94.92 & 97.24 & 97.39 & 96.69 & 95.59 \\
\hline & 0.4 & 86.66 & 88.94 & 92.14 & 95.13 & 96.60 & 97.47 & 96.95 \\
\hline & 0.5 & 89.02 & 91.38 & 93.92 & 94.86 & 94.78 & 97.23 & 99.41 \\
\hline & 0.6 & 95.40 & 97.29 & 97.18 & 93.64 & 91.91 & 94.83 & 100.96 \\
\hline & 0.7 & 98.69 & 98.73 & 96.98 & 91.14 & 88.82 & 92.16 & 99.05 \\
\hline & 0.8 & 99.02 & 98.80 & 95.27 & 88.93 & 86.67 & 90.63 & 98.26 \\
\hline \multirow{7}{*}{ CCSS } & 0.2 & 99.03 & 98.82 & 95.03 & 88.84 & 86.67 & 90.65 & 98.33 \\
\hline & 0.3 & 98.70 & 98.68 & 96.83 & 91.07 & 88.82 & 92.17 & 99.06 \\
\hline & 0.4 & 95.40 & 97.30 & 97.09 & 93.59 & 91.92 & 94.84 & 100.99 \\
\hline & 0.5 & 89.01 & 91.37 & 93.87 & 94.82 & 94.96 & 97.25 & 99.42 \\
\hline & 0.6 & 86.65 & 88.93 & 92.10 & 95.10 & 96.61 & 97.47 & 96.93 \\
\hline & 0.7 & 90.63 & 92.21 & 94.89 & 97.22 & 97.39 & 96.69 & 95.57 \\
\hline & 0.8 & 98.26 & 99.07 & 100.96 & 99.30 & 96.95 & 95.63 & 95.03 \\
\hline \multirow{7}{*}{ CSCS } & 0.2 & 98.68 & 99.70 & 96.60 & 94.59 & 96.60 & 99.70 & 98.68 \\
\hline & 0.3 & 100.35 & 100.95 & 99.60 & 98.53 & 99.60 & 100.95 & 100.35 \\
\hline & 0.4 & 100.63 & 101.43 & 100.62 & 99.97 & 100.62 & 101.43 & 100.62 \\
\hline & 0.5 & 100.75 & 101.79 & 101.07 & 100.45 & 101.07 & 101.79 & 100.75 \\
\hline & 0.6 & 100.63 & 101.43 & 100.62 & 99.97 & 100.62 & 101.43 & 100.62 \\
\hline & 0.7 & 100.35 & 100.95 & 99.60 & 98.53 & 99.60 & 100.95 & 100.35 \\
\hline & 0.8 & 98.68 & 99.70 & 96.60 & 94.59 & 96.60 & 99.70 & 98.68 \\
\hline
\end{tabular}


Table 7: Continued.

\begin{tabular}{|c|c|c|c|c|c|c|c|c|}
\hline \multirow{2}{*}{ Edge condition } & \multirow{2}{*}{$\bar{y}$} & \multicolumn{7}{|c|}{$\bar{x}$} \\
\hline & & 0.2 & 0.3 & 0.4 & 0.5 & 0.6 & 0.7 & 0.8 \\
\hline \multirow{7}{*}{ SCSC } & 0.2 & 98.88 & 100.37 & 100.64 & 100.83 & 100.64 & 100.37 & 98.88 \\
\hline & 0.3 & 99.76 & 101.04 & 101.52 & 101.86 & 101.53 & 101.04 & 99.75 \\
\hline & 0.4 & 96.65 & 99.74 & 100.713 & 101.14 & 100.76 & 99.74 & 96.64 \\
\hline & 0.5 & 94.69 & 98.67 & 100.09 & 100.53 & 100.09 & 98.67 & 94.69 \\
\hline & 0.6 & 96.64 & 99.74 & 100.76 & 101.14 & 100.76 & 99.74 & 96.64 \\
\hline & 0.7 & 99.76 & 101.04 & 101.53 & 101.86 & 101.52 & 101.04 & 99.75 \\
\hline & 0.8 & 98.88 & 100.37 & 100.64 & 100.83 & 100.64 & 100.37 & 98.88 \\
\hline \multirow{7}{*}{ CSSS } & 0.2 & 97.88 & 98.69 & 96.54 & 93.98 & 94.57 & 97.61 & 98.39 \\
\hline & 0.3 & 99.43 & 99.86 & 99.39 & 98.38 & 98.64 & 99.79 & 99.86 \\
\hline & 0.4 & 99.76 & 100.55 & 100.55 & 100.23 & 100.53 & 101.37 & 101.32 \\
\hline & 0.5 & 100.33 & 101.13 & 100.94 & 100.64 & 101.07 & 102.09 & 101.97 \\
\hline & 0.6 & 99.76 & 100.55 & 100.55 & 100.23 & 100.52 & 101.54 & 101.32 \\
\hline & 0.7 & 99.43 & 99.86 & 99.39 & 98.38 & 98.64 & 99.80 & 99.86 \\
\hline & 0.8 & 97.88 & 98.68 & 96.54 & 93.98 & 94.57 & 97.61 & 98.39 \\
\hline \multirow{7}{*}{ SSSC } & 0.2 & 98.24 & 99.78 & 101.36 & 102.07 & 101.36 & 99.77 & 98.24 \\
\hline & 0.3 & 97.52 & 99.76 & 101.40 & 102.14 & 101.40 & 99.76 & 97.51 \\
\hline & 0.4 & 94.58 & 98.68 & 100.53 & 101.03 & 100.53 & 98.68 & 94.58 \\
\hline & 0.5 & 94.08 & 98.44 & 100.24 & 100.63 & 100.24 & 98.44 & 94.08 \\
\hline & 0.6 & 96.58 & 99.47 & 100.62 & 100.96 & 100.62 & 99.47 & 96.57 \\
\hline & 0.7 & 98.72 & 99.90 & 100.61 & 101.15 & 100.61 & 99.90 & 98.72 \\
\hline & 0.8 & 98.03 & 99.41 & 99.73 & 100.34 & 99.72 & 99.41 & 98.03 \\
\hline \multirow{7}{*}{ SSCS } & 0.2 & 98.39 & 97.61 & 94.57 & 93.99 & 96.55 & 98.69 & 97.88 \\
\hline & 0.3 & 99.87 & 99.80 & 98.65 & 98.39 & 99.40 & 99.87 & 99.43 \\
\hline & 0.4 & 101.32 & 101.38 & 100.53 & 100.23 & 100.55 & 100.56 & 99.76 \\
\hline & 0.5 & 101.98 & 102.10 & 101.07 & 100.64 & 100.95 & 101.13 & 100.33 \\
\hline & 0.6 & 101.33 & 101.38 & 100.53 & 100.23 & 100.55 & 100.56 & 99.76 \\
\hline & 0.7 & 99.87 & 99.80 & 98.65 & 98.39 & 99.40 & 99.87 & 99.43 \\
\hline & 0.8 & 98.39 & 97.62 & 94.58 & 93.99 & 96.54 & 98.69 & 97.88 \\
\hline \multirow{7}{*}{ SSSS } & 0.2 & 97.32 & 97.03 & 111.45 & 93.16 & 94.72 & 97.03 & 97.32 \\
\hline & 0.3 & 96.96 & 98.53 & 98.61 & 98.09 & 98.62 & 98.53 & 96.96 \\
\hline & 0.4 & 94.66 & 98.58 & 100.57 & 100.43 & 100.57 & 98.58 & 94.65 \\
\hline & 0.5 & 93.19 & 98.05 & 100.34 & 100.80 & 100.34 & 98.05 & 93.18 \\
\hline & 0.6 & 94.65 & 98.58 & 100.57 & 100.43 & 100.57 & 98.58 & 94.65 \\
\hline & 0.7 & 96.95 & 98.53 & 98.61 & 98.09 & 98.62 & 98.53 & 96.96 \\
\hline & 0.8 & 97.31 & 97.03 & 94.72 & 93.16 & 94.72 & 97.03 & 97.32 \\
\hline
\end{tabular}

$a / b=1, a / h=100, a^{\prime} / b^{\prime}=1, c / a=0.2 ; E_{11} / E_{22}=25, G_{23}=0.2 E_{22}, G_{13}=G_{12}=0.5 E_{22}, \nu_{12}=v_{21}=0.25$. 
From the $r$ values it is observed that a shell with eccentric cutout has a fundamental frequency above $90 \%$ to that of a shell having concentric cutout in almost all the cases of 0/90/0/90 shells. So cutout centre may be moved along any directions, resulting in loss of frequency of not more than $5-10 \%$ with respect to a shell with concentric cutout. But in case of a $+45 /-45 /+45 /-45$ shell, the $r$ value is between 84.02 and 102.10 .

For 0/90/0/90 shell when the four edges are clamped the stiffness of shell decreases more when the cutout centre is nearer to the shell centre line whereas when the cutout centre goes towards the boundary shell stiffness increases resulting in increased frequency. For such a shell $r$ value is minimum along centre line and increases towards the boundary. But such increase or decrease is marginal in each case. When one simply supported edge is introduced along the shell boundary $r$ value is maximum along the simply supported boundary in almost all the cases. Similarly when two adjacent edges are simply supported the values of $r$ have comparatively greater value towards simply supported edges. But exactly reverse trend is observed when two opposite edges are simply supported. In such cases the maximum $r$ values are obtained along the centre line of the shell which is equidistant from each simply supported edge and $r$ value decreases towards the simply supported edges. It is further noticed that when three edges are simply supported the $r$ value is maximum along the line which is equidistant from two simply supported edges and $r$ value is minimum along the centre which is equidistant from one simply supported edge and another clamped edge. When four edges are simply supported $r$ value is maximum along the diagonal.

In case of $+45 /-45 /+45 /-45$ shells when the four edges are clamped the $r$ values are maximum along the centre line of shell in both directions but towards the boundary no unified trend is observed. Also along each clamped edge $r$ value is maximum at the middle of each edge. When one of the edges is simply supported, the $r$ value decreases towards the edges opposite to the simply supported edges and are minimum at the centre of that edge. But along the simply supported edge and other two clamped edges fundamental frequency is maximum at the middle of each edge. When the two adjacent edges are simply supported and other two are clamped minimum $r$ values are obtained when cutout centre is near the middle of the clamped edges and maximum when the same is near the middle of the simply supported edges. When two opposite edges are simply supported in more than $50 \%$ cases $r$ value is greater than 100 . In rest of the cases $r$ values are more than 95 except one or two cases. When three edges are simply supported in a central rectangular zone the $r$ value is greater than 100. But when four edges are simply supported the zone where $r$ value is greater than 100 gets reduced.

\section{Conclusions}

From the present study the following conclusions are drawn.

(1) The finite element code used here is suitable for analyzing free vibration problems of hypar shell roofs with cutouts as this approach produces results in close agreement with those of the benchmark problems.

(2) Concentric cutouts may be provided safely on hypar shell surfaces for functional requirements up to $a^{\prime} / a=0.2$.

(3) The arrangement of boundary constraint along the four edges is far more important than their actual number so far the free vibration stiffness is concerned.

(4) Fundamental frequency undergoes marked improvement when the edge is converted to clamped from simply supported condition.

(5) Tables 4 and 5 provide a clear picture about the relative free vibration performances of hypar shells for different combinations of edge conditions along the four sides and are expected to be very useful in decision making for practicing engineers.

(6) Tables 6 and 7 provide the information regarding behaviour of hypar shell with eccentric cutouts for wide spectrum of eccentricity and boundary conditions and may be used as design aids by structural engineers.

\section{Notations}

$\begin{array}{ll}a, b: & \text { Length and width of shell in plane } \\ a^{\prime}, b^{\prime}: & \text { Length and width of cutout in plane } \\ c: & \text { Rise of hypar shell } \\ \left\{d_{e}\right\}: & \text { Element displacement } \\ E_{11}, E_{22}: & \text { Elastic moduli } \\ G_{12}, G_{13}, G_{23}: & \text { Shear moduli of a lamina with respect to } \\ & 1,2 \text { and } 3 \text { axes of fibre } \\ h: & \text { Shell thickness } \\ M_{x}, M_{y}: & \text { Moment resultants } \\ M_{x y}: & \text { Torsion resultant } \\ \text { np: } & \text { Number of plies in a laminate } \\ N_{1}-N_{8}: & \text { Shape functions } \\ N_{x}, N_{y}: & \text { Inplane force resultants } \\ N_{x y}: & \text { Inplane shear resultant } \\ Q_{x}, Q_{y}: & \text { Transverse shear resultant } \\ R_{x y}: & \text { Radii of cross curvature of hypar shell } \\ u, v, w: & \text { Translational degrees of freedom } \\ x, y, z: & \text { Local co-ordinate axes } \\ X, Y, Z: & \text { Global co-ordinate axes } \\ z_{k}: & \text { Distance of bottom of the } k \text { th ply from } \\ \alpha, \beta: & \text { midsurface of a laminate } \\ \varepsilon_{x}, \varepsilon_{y}: & \text { Rotational degrees of freedom } \\ \phi: & \text { Inplane strain component } \\ \gamma_{x y}, \gamma_{x z}, \gamma_{y z}: & \text { Angle of twist } \\ \nu_{12}, \nu_{21}: & \text { Poisson's ratios } \\ \xi, \eta, \tau: & \text { Isoparametric coordinates } \\ \rho: & \text { Density of material } \\ \sigma_{x}, \sigma_{y}: & \text { Inplane stress components } \\ \tau_{x y}, \tau_{x z}, \tau_{y z}: & \text { Shearing stress components } \\ & \end{array}$


$\omega:$ Natural frequency

$\bar{\omega}$ : Non-dimensional natural frequency

$$
=\omega a^{2}\left(\rho / E_{22} h^{2}\right)^{1 / 2} \text {. }
$$

\section{References}

[1] D. Chakravorty, P. K. Sinha, and J. N. Bandyopadhyay, "Applications of FEM on free and forced vibration of laminated shells," Journal of Engineering Mechanics, vol. 124, no. 1, pp. $1-8,1998$.

[2] J. N. Reddy, "Large amplitude flexural vibration of layered composite plates with cutouts," Journal of Sound and Vibration, vol. 83, no. 1, pp. 1-10, 1982.

[3] S. K. Malhotra, N. Ganesan, and M. A. Veluswami, "Vibration of composite plates with cut-outs," Journal of Aeronautical Society of India, vol. 41, pp. 61-64, 1989.

[4] B. Sivasubramonian, A. M. Kulkarni, G. Venkateswara Rao, and A. Krishnan, "Free vibration of curved panels with cutouts," Journal of Sound and Vibration, vol. 200, no. 2, pp. 227-234, 1997.

[5] K. Sivakumar, N. G. R. Iyengar, and K. Deb, "Free vibration of laminated composite plates with cutout," Journal of Sound and Vibration, vol. 221, no. 3, pp. 443-465, 1999.

[6] R. E. Rossi, "Transverse vibrations of thin, orthotropic rectangular plates with rectangular cutouts with fixed boundaries," Journal of Sound and Vibration, vol. 221, no. 4, pp. 733-736, 1999.

[7] M. Huang and T. Sakiyama, "Free vibration analysis of rectangular plates with variously-shaped holes," Journal of Sound and Vibration, vol. 226, no. 4, pp. 769-786, 1999.

[8] S. S. Hota and P. Padhi, "Vibration of plates with arbitrary shapes of cutouts," Journal of Sound and Vibration, vol. 302, no. 4-5, pp. 1030-1036, 2007.

[9] B. Sivasubramonian, G. V. Rao, and A. Krishnan, "Free vibration of longitudinally stiffened curved panels with cutout," Journal of Sound and Vibration, vol. 226, no. 1, pp. 41-55, 1999.

[10] S. S. Hota and D. Chakravorty, "Free vibration of stiffened conoidal shell roofs with cutouts," JVC/Journal of Vibration and Control, vol. 13, no. 3, pp. 221-240, 2007.

[11] N. Nanda and J. N. Bandyopadhyay, "Nonlinear free vibration analysis of laminated composite cylindrical shells with cutouts," Journal of Reinforced Plastics and Composites, vol. 26, no. 14, pp. 1413-1427, 2007.

[12] M. S. Qatu, Vibration of Laminated Shells and Plates, Elsevier, London, UK, 2004.

[13] V. V. Vasiliev, R. M. Jones, and L. I. Man, Mechanics of Composite Structures, Taylor \& Francis, Boca Raton, Fla, USA, 1993.

[14] A. Dey, J. N. Bandyopadhyay, and P. K. Sinha, "Finite element analysis of laminated composite paraboloid of revolution shells," Computers and Structures, vol. 44, no. 3, pp. 675-682, 1992.

[15] R. D. Cook, D. S. Malkus, and M. E. Plesha, Concepts and Applications of Finite Element Analysis, John Wiley \& Sons, New York, NY, USA, 1989.

[16] S. Sahoo and D. Chakravorty, "Finite element vibration characteristics of composite hypar shallow shells with various edge supports," JVC/Journal of Vibration and Control, vol. 11, no. 10, pp. 1291-1309, 2005. 

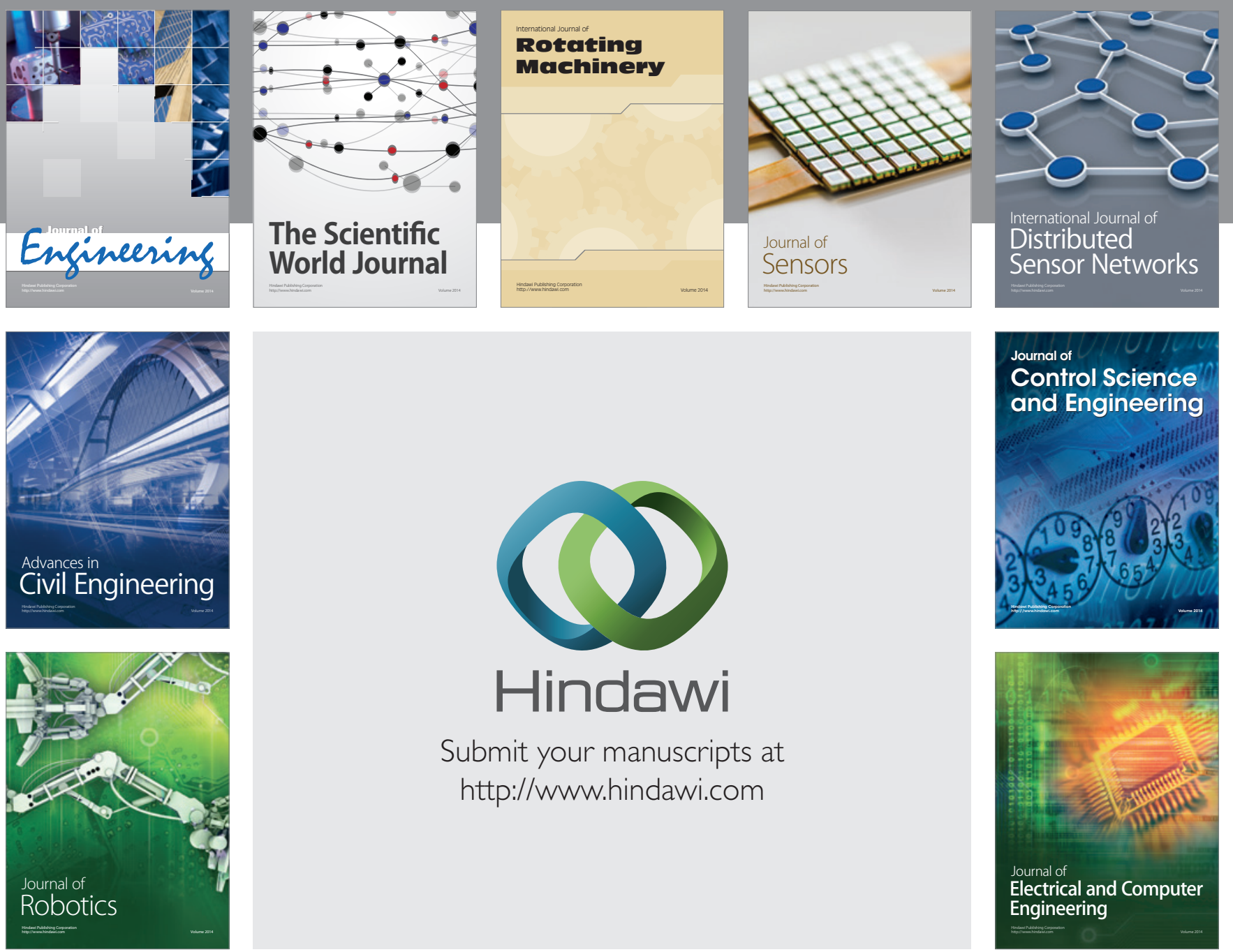

Submit your manuscripts at

http://www.hindawi.com
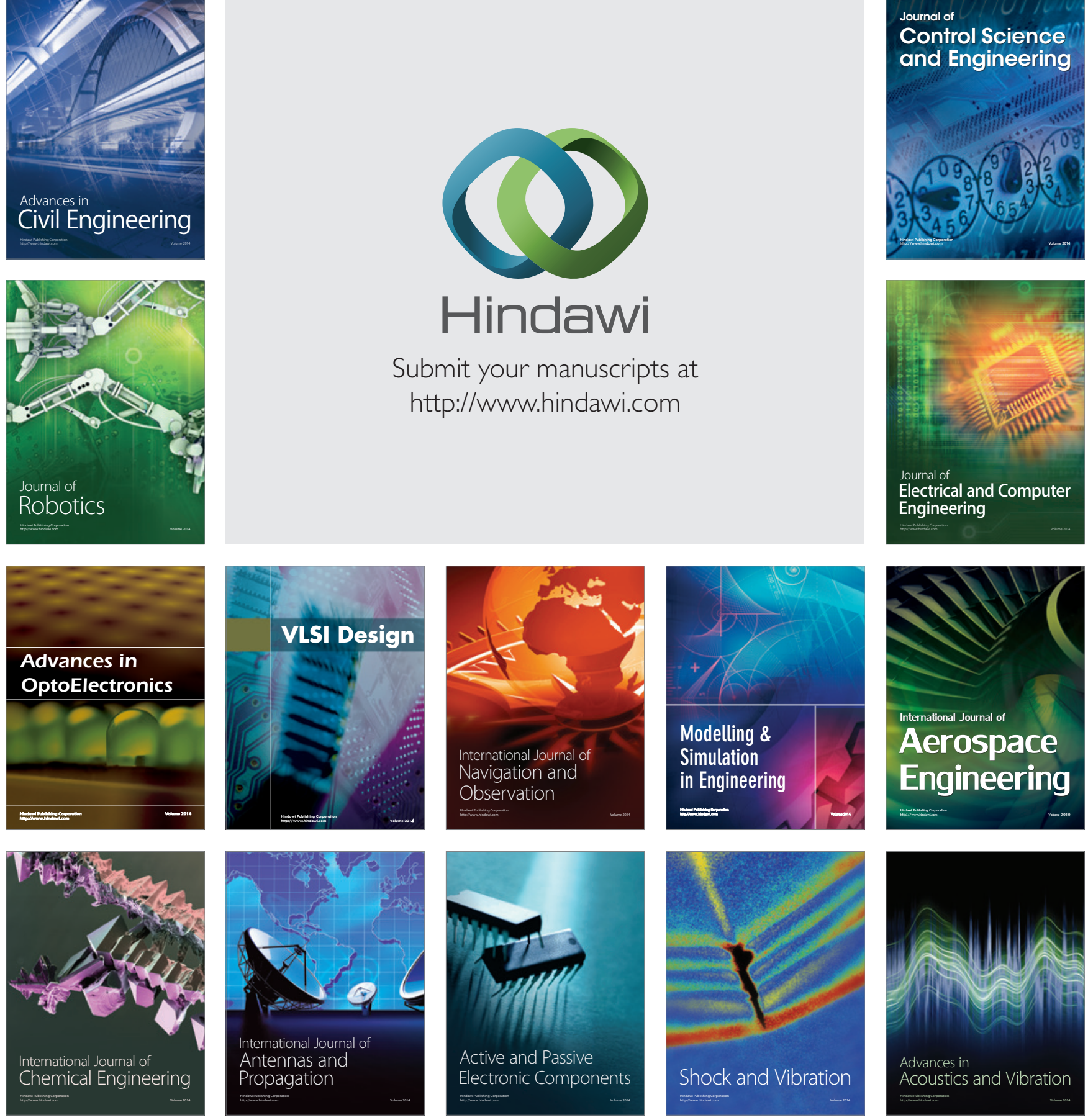